\title{
Latitudinal dependence of the solar wind density derived from remote sensing measurements using interplanetary Lyman $\alpha$ emission from 1999 to 2002
}

\author{
Hiromu Nakagawa ${ }^{1}$, Hiroshi Fukunishi ${ }^{2}$, Shigeto Watanabe ${ }^{3}$, Yukihiro Takahashi ${ }^{1}$, Makoto Taguchi $^{4}$, and Rosine Lallement ${ }^{5}$ \\ ${ }^{1}$ Department of Geophysics, University of Tohoku, Sendai, Japan \\ ${ }^{2}$ Japan Society for the Promotion of Science, Beijing, China \\ ${ }^{3}$ Department of Earth and Planetary Science, University of Hokkaido, Sapporo, Japan \\ ${ }^{4}$ Department of Physics, College of Science, Rikkyo University, Tokyo, Japan \\ ${ }^{5}$ Service d'Aéronomie, Verrières le Buisson, France
}

(Received July 31, 2007; Revised October 1, 2008; Accepted October 15, 2008; Online published March 3, 2009)

\begin{abstract}
The interplanetary Lyman $\alpha$ backscattered emission is an effective tool for remote sensing of the global structure of the solar wind proton flux. This paper reports an attempt to derive the latitudinal dependence of the solar wind density by combining the interplanetary Lyman $\alpha$ measurements of the Nozomi spacecraft for the period 1999-2002 with the solar wind speed data derived from interplanetary scintillation measurements. This approach successfully revealed the slow and dense solar wind over the poles during the period of the solar maximum. Data on the polar solar wind density indicate a significant growth from the middle of 2000 , and the polar values of solar wind density are close to those of the equatorial values as a result of the disappearance of the coronal hole. A marked density depletion occurred in the middle of 2001, which can be ascribed to the development of fast winds from the polar coronal hole. To evaluate the remote sensing method, we considered solar wind density data from in situ measurements obtained by the Ulysses spacecraft. We conclude that our method basically agrees with in situ measurements, although we found a significant (a factor of 2) difference between these in the middle of 2001.
\end{abstract}

Key words: Sun, solar wind, interplanetary neutral hydrogen, heliosphere, Nozomi.

\section{Introduction}

The Sun is located in a warm $(\sim 6500 \mathrm{~K})$ and partly ionized cloud of interstellar gas, called the local interstellar cloud (LIC), and the heliosphere surrounding the Sun is formed by the interaction between the expanding solar wind and the components of the LIC (Fig. 1). This interaction has a complex structure in which the solar wind, interstellar neutral atoms, galactic and anomalous cosmic rays, and pickup ions all play roles (Fahr, 1996; Zank, 1999; Fahr et al., 2000; Izmodenov, 2004; Baranov, 2006; Izmodenov and Baranov, 2006). Based on a great number of space- and ground-based observations over the century, significant advances have been booked in our understanding of the interaction between the three-dimensional heliosphere and the ambient interstellar medium. Although these achievements have spurred the development of increasingly sophisticated models attempting to describe various aspects of the physics underlying the interaction between the solar wind and the interstellar medium (Fahr, 1996; Zank, 1999; Fahr et al., 2000; Izmodenov, 2004; Baranov, 2006; Izmodenov and Baranov, 2006), to our knowledge no direct observations of the different components of the LIC within the heliospheric interface and beyond have been reported to date. It is also difficult to understand the global structure of the

Copyright (c) The Society of Geomagnetism and Earth, Planetary and Space Sciences (SGEPSS); The Seismological Society of Japan; The Volcanological Society of Japan; The Geodetic Society of Japan; The Japanese Society for Planetary Sciences; TERRAPUB heliosphere using one-point measurements.

The global three-dimensional structure of the solar wind is little understood owing to the shortage of observational data-with the exception of the recent in situ observations of the Ulysses spacecraft (e.g., Phillips et al., 1995; Marsden et al., 1996; McComas et al., 2002, 2003) and interplanetary scintillation (IPS) observations that indicated the changes in the global structure of the solar wind speed according to the solar activity cycle (e.g., Kojima and Kakinuma, 1987, 1990).

Observations of the interplanetary Lyman $\alpha$ emission backscattered resonantly by interplanetary neutral hydrogen atoms are useful for improving our knowledge of the solar wind out of the ecliptic plane, since these provide reliable information on the latitudinal dependence of the solar wind proton flux in the inner heliosphere. Joselyn and Holzer (1975) first determined the latitudinal dependence of the solar wind proton flux using backscattered interstellar hydrogen Lyman $\alpha$ emission. Several subsequent attempts have been made to investigate the global structure of the solar wind proton flux from interplanetary Lyman $\alpha$ observations based on the concept that the interplanetary Lyman $\alpha$ emissions observed in the inner heliosphere are influenced mainly by the ionization due to charge exchange with solar wind protons. The ionization data obtained from these studies indicate that the solar wind drastically changes its global structure during the solar activity cycle (e.g., Bertaux et al., 1995; Summanen, 2000; Nakagawa et al., 2003; Pryor et al., 2003; Quémerais et al., 2006). Kumar and Broadfoot 


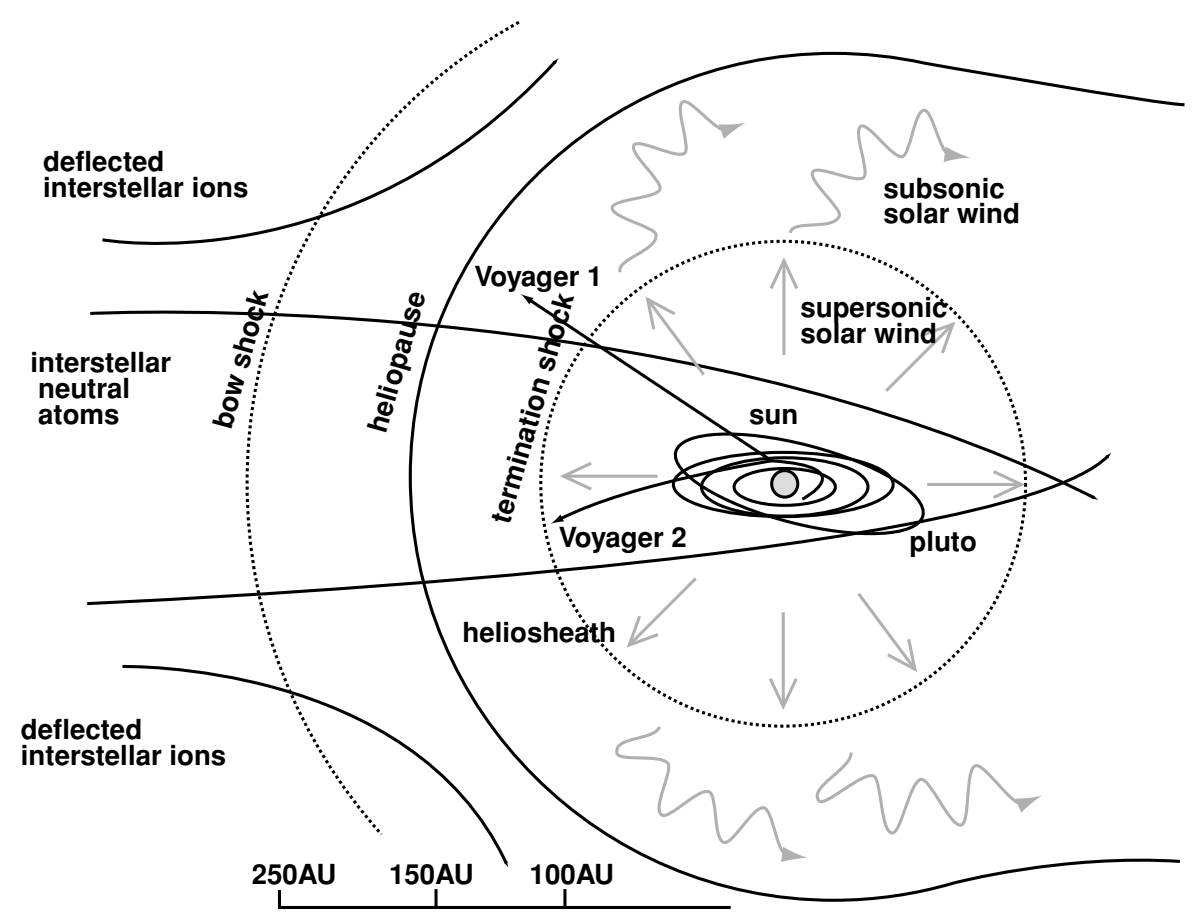

Fig. 1. Schematic drawing of the heliosphere, which is formed by the interaction between the expanding solar wind and the local interstellar cloud (LIC).

(1979) and Lallement et al. (1985) predicted the presence of large decreases in solar wind proton flux from the ecliptic plane toward the poles. Kyrölä et al. (1998) estimated that the reduction of the particle flux from the equator to the poles is $18 \%$ on average at the solar minimum. Nakagawa et al. (2003) have shown that during the ascending to the maximum activity phase, the total ionization rate of interstellar hydrogen becomes gradually isotropic over almost the entire range of heliolatitudes. Recently, Quémerais et al. (2006) derived the latitudinal dependence of the ionization rate over a 10 -year period using observations obtained by the solar wind anisotropy instrument (SWAN) at the Solar and Heliospheric Observatory (SOHO) and computed the Lyman $\alpha$ distribution using the hot model (Lallement et al., 1985). Their results clearly show that the anisotropic ionization rate pattern changes to an isotropic pattern as the solar activity increases. In the solar maximum phase, the polar values of the ionization rate are very close to the equatorial values, and the isotropic pattern can be ascribed to the uniform distribution at the solar maximum phase.

Although the latitudinal dependence of the ionization rate and the variations in its solar cycle have been well investigated, the conversion of ionization rate data to solar wind density has not yet been attempted. The main purpose of this study was to investigate the latitudinal dependence of the solar wind density and its long-term variation (3 years) around the solar maximum using Lyman $\alpha$ emission data, with the aim of gaining an understanding of the three-dimensional structure of the solar wind parameters and evaluating the remote sensing method. We derived the solar wind density from a comprehensive study that focused on Lyman $\alpha$ data obtained from Nozomi spacecraft and solar wind speed data obtained from IPS observations by the Solar-Terrestrial Environment Laboratory (STEL) of

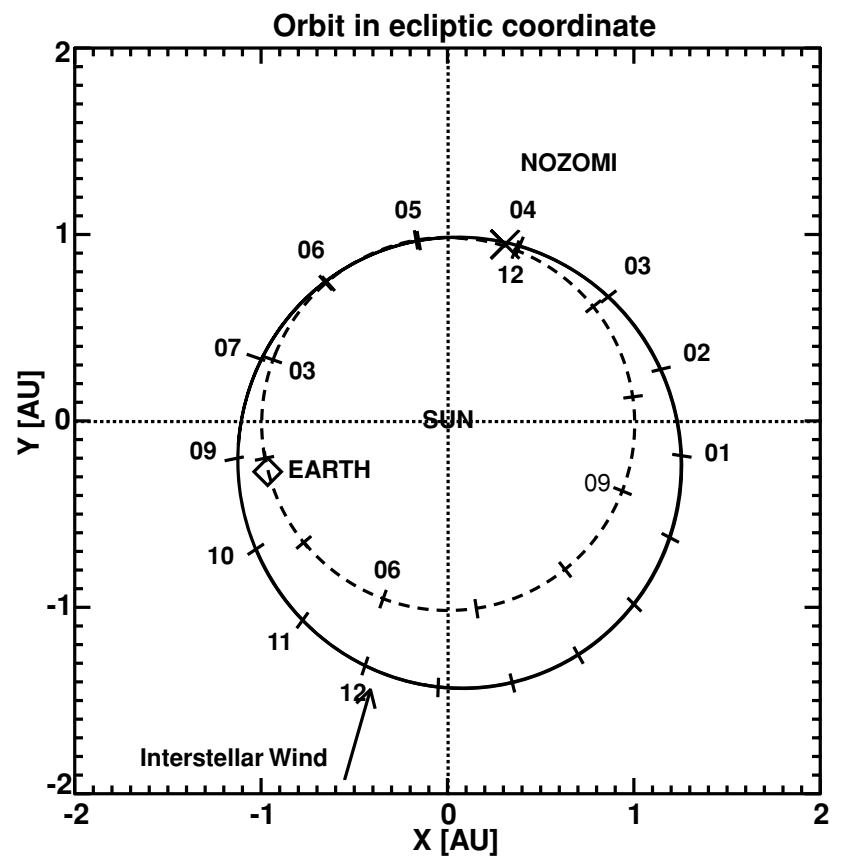

Fig. 2. Ecliptic plane projections of the orbits of Nozomi (solid line) and Earth (dashed line). The numbers on the lines represent the months during 2000. The cross marks the position of the spacecraft and the diamond that of the Earth on April 5, 2000, corresponding to Figs. 3 and 4. The line of sight of the Lyman $\alpha$ observations discussed in the text are almost always perpendicular to the line connecting the Nozomi spacecraft and Earth. Note that the orbit of Nozomi is inclined at a small angle to the ecliptic plane.

Nagoya University (Kojima et al., 1998). We also compared the derived density data with in situ measurements obtained by the Ulysses spacecraft. In Sections 2-6, we describe the remote sensing methods used for deriving the 
LOS in ecliptic coordinates

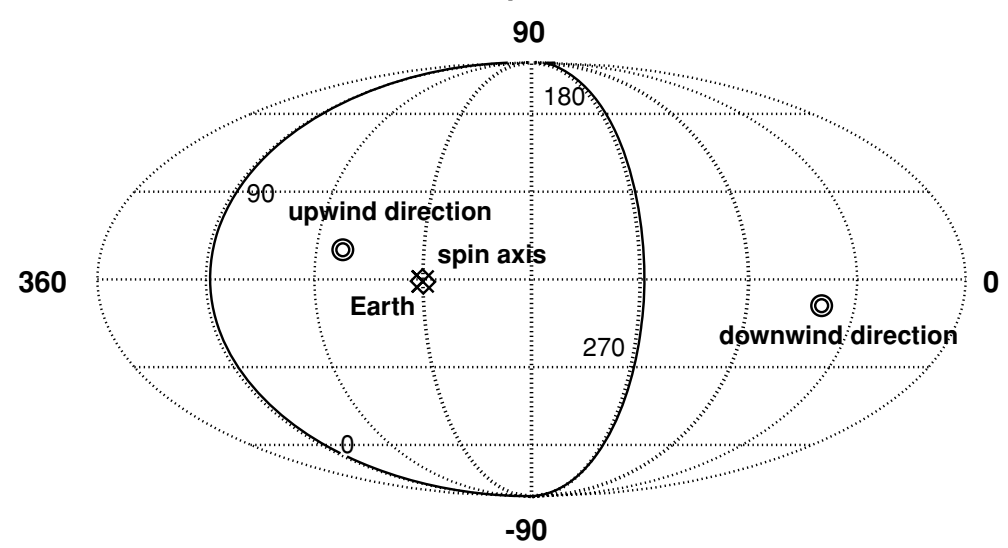

Fig. 3. Circular tracks of the lines of sight of the Nozomi UVS (solid) instrument on April 5, 2000, projected on a sky map. The directions of the spin axis and Earth are shown by the cross and the diamond, respectively, and are practically identical. The upwind and downwind directions of the interstellar neutral hydrogen are shown by the double circles. The numbers at the line of sight contour of UVS correspond to the spin angle.

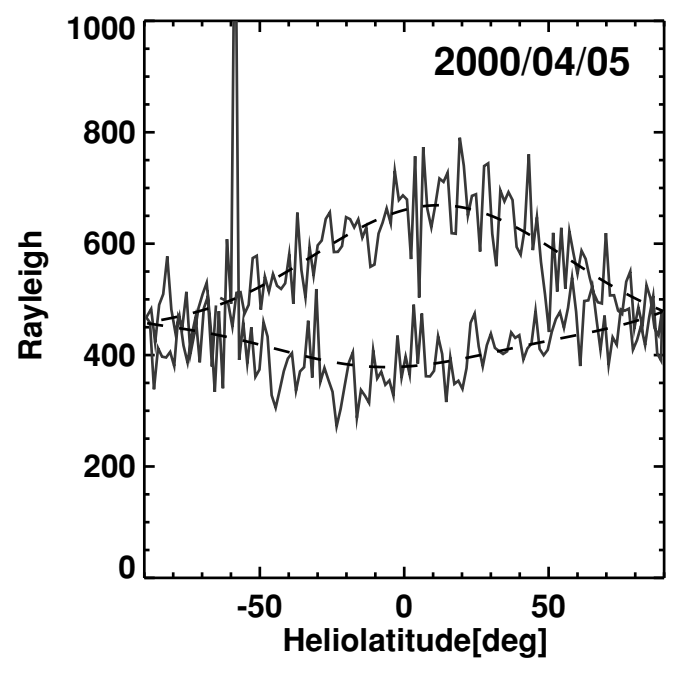

Fig. 4. The intensity of the Lyman $\alpha$ emission observed by the UVS instrument on April 5, 2000 that corresponds to the Fig. 3. The units of the intensity are Rayleighs. The solid line shows the original data, and the broken line shows the data corrected by low- and medium-pass filtering, both of which reduce noise and the star contamination seen, for example, at $-59^{\circ}$ in the heliographic latitude.

latitudinal dependence of the solar wind density. The latitudinal dependence of the solar wind density derived here is discussed in Section 7, and the solar wind density derived from our method is compared with that from the Ulysses measurements in Section 8. Our conclusions are presented in Section 9.

\section{Observations}

The Nozomi spacecraft was in the Mars transfer orbit with perihelion at $1 \mathrm{AU}$ and aphelion at $1.5 \mathrm{AU}$ (Fig. 2). The observations were performed during the cruise period in the Mars transfer orbit. Nozomi is a spin-stabilized spacecraft with a high gain antenna fixed to its top. Its spin axis was always directed toward the Earth. The Lyman $\alpha$ emission of neutral interstellar hydrogen at $121.6 \mathrm{~nm}$ was recorded by the ultraviolet imaging spectrometer (UVS) instrument from March 1999 until April 2002.

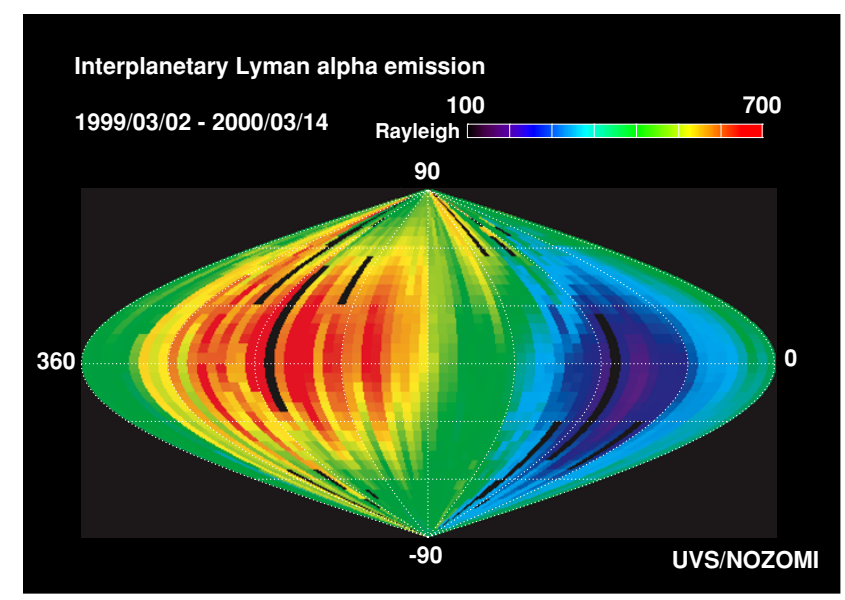

Fig. 5. Full sky intensity map of the interplanetary hydrogen Lyman $\alpha$ emission as derived from UVS instrument performed during 1 year, from March 2, 1999 to March 14, 2000. The intensities are averaged in each $5^{\circ} \times 5^{\circ}$ grid.

The UVS instrument has two sensors: a grating spectrometer (UVS-G), operating at wavelengths between 110 and $310 \mathrm{~nm}$ with a spectral resolution of 2-3 nm, and a hydrogen and deuterium absorption cell photometer (UVS-P) (Fukunishi et al., 1999). In this study, the H Lyman $\alpha$ emission intensity at $121.6 \mathrm{~nm}$ was derived from the UVS-G data integrated over 110-130 nm in the period from March 2, 1999 to April 24, 2002. The field of view (FOV) of UVS-G is perpendicular to the spin axis of the spacecraft directed towards the Earth, which allows the UVS instrument to capture a full sky image during half of the revolution about the Sun using the spin and orbital motion of the spacecraft. The FOV in the plane perpendicular to the axis is equal to $0.09^{\circ}$ and in the plane including the spin axis, it is $0.29^{\circ}$. The spin period (normally $8 \mathrm{~s}$ ) is divided into 256 intervals for data sampling. The spatial resolution of UVS is therefore $1.41^{\circ}$ in the plane perpendicular to the spin axis and $0.29^{\circ}$ in the plane including the spin axis. An example track of the line of sight (LOS) of UVS on April 5, 2000 is shown in Fig. 3 as a solid-line oval, and the observations obtained from this 
Table 1. Values of parameters used for the hot model of hydrogen.

\begin{tabular}{lll}
\hline Parameters & Values & Reference \\
\hline Density of hydrogen at infinity & $0.11\left(\mathrm{~cm}^{-3}\right)$ & Gloeckler and Geiss (2001) \\
Velocity of hydrogen at infinity & $22.0(\mathrm{~km} / \mathrm{s})$ & Lallement et al. $(2005)$ \\
Temperature of hydrogen at infinity & $11500(\mathrm{~K})$ & Lallement et al. $(2005)$ \\
Upwind direction of hydrogen & $252.5^{\circ}, 8.8^{\circ}$ & Lallement et al. $(2005)$ \\
\hline
\end{tabular}

scan are shown in Fig. 4. One of the full-sky Lyman $\alpha$ intensity maps, obtained by the UVS instrument during an approximately 1-year interval from March 2, 1999 to March 14, 2000, is shown in Fig. 5.

As shown in Fig. 3, each latitudinal scan of the sky is yielded in the upwind and downwind hemisphere. The scan in the upwind hemisphere is collected from opposite directions in the hemisphere to the other scan in the downwind hemisphere. The scans can also be plotted as a function of ecliptic latitude, as in Fig. 4. For the analysis presented in this paper, we selected data from lines of sight located in the upwind hemisphere. Details of the UVS instrumentation and the method used to construct full-sky images from the UVS latitudinal scans are described by Taguchi et al. (2000).

\section{Calculations with the Hot Model}

Interpretation of the observations was carried out with the use of a hot model of neutral interstellar gas distribution in the heliosphere. This model has been described by Fahr (1978), Thomas (1978), Wu and Judge (1979), and Lallement et al. (1985), among others. In this study, a hot model based on the investigations carried out by $\mathrm{Wu}$ and Judge (1979) and Lallement et al. (1985) was used to calculate the interplanetary neutral hydrogen distribution along the LOS of the instrument. In this model, the Maxwell-Boltzman distribution is assumed for the velocity distribution of hydrogen atoms at infinity, i.e., where the solar influence can be neglected but where it is still inside the heliospheric interface, characterized by the density $n$, temperature $T$, and the bulk velocity $V$ of the unperturbed gas. It should be noted that the existence of the heliospheric interface and hydrogen-proton coupling by charge exchange can induce significant changes between the local conditions following crossing of the interface region and the actual conditions of the interstellar gas, as shown by Zank (1999), Müller et al. (2004), and Pogorelov et al. (2004). However, closer to the Sun, both models describe the hydrogen atoms in a similar manner.

Radiation pressure compensates for the solar gravity to an extent depending on the intensity of solar radiation in the Lyman $\alpha$ line and the trajectories of hydrogen atoms are hyperbolic, with eccentricities and semi major axes determined by the ratio $\mu$ of solar radiation pressure to solar gravity. The ionization rate of the interplanetary hydrogen $\beta$, which is a measure of the loss process in the neutral hydrogen atoms on its approach to the Sun, was expressed as a function of heliographic latitude $b$ and distance from the Sun $r$. The ionization rate was calculated at each point along the path of a hydrogen atom during its travel through the solar system. The probability of survival of the atom

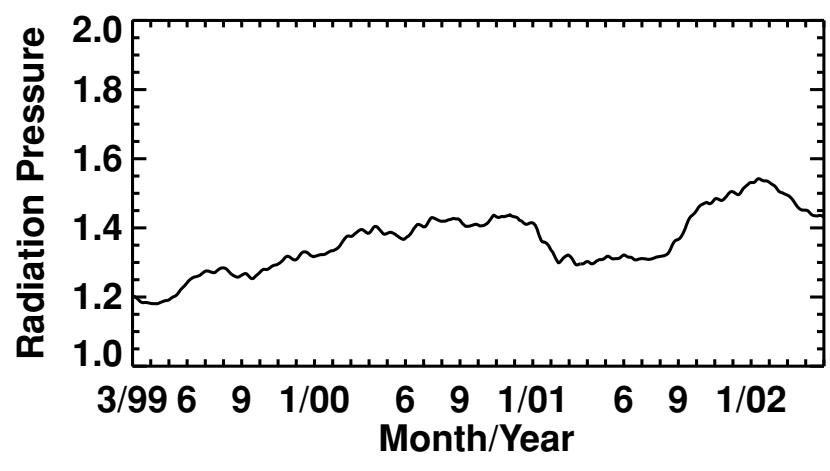

Fig. 6. Time variations in solar radiation pressure normalized by solar gravity, as used in the hot model. Monthly averages are computed for each moment of the Nozomi observations from a time series of the solar Lyman $\alpha$ flux returned by the SOLAR2000 model.

until it reaches a given point in space was calculated by integrating the ionization rate probability over the entire path from infinity to the given point.

The parameters of the gas at infinity were adopted as follows: the upwind direction of interstellar neutral hydrogen was adopted as determined based on the SOHO/SWAN observations by Lallement et al. (2005) $\left(252.5^{\circ}, 8.8^{\circ}\right)$ in the ecliptic coordinates, and the bulk velocity $(22.0 \mathrm{~km} / \mathrm{s})$ and temperature $(11500 \mathrm{~K})$ based on also Lallement et al. (2005). The density $\left(0.11 \mathrm{~cm}^{-3}\right)$ was adopted as determined based on Ulysses in situ observations of interstellar hydrogen by Gloeckler and Geiss (2001). The parameters used in the model are summarized in Table 1.

The most important feature of this calculation is that both the ionization rates and radiation pressure are based on actual observations. Figure 6 shows the monthly averages of radiation pressure expressed in units of solar gravity, computed for each moment of the Nozomi observations. These monthly averages are used to compute the simulated intensities corrected for fluctuations of the solar illumination. The model is fitted to the Lyman $\alpha$ time series obtained from SOLAR2000 (Tobiska et al., 2000). For simplification, the solar line profile was assumed to be flat, so that the radiation pressure does not depend on the radial velocity of the atoms. In reality, the solar line profile is not flat and corresponds to approximately a ca. $10 \%$ change in neutral hydrogen density.

The ionization processes of the interplanetary hydrogen are simulated as a sum of the charge-exchange of hydrogen atoms with solar wind protons, photoionization, and electron impact, as summarized by Bzowski et al. (2008). Here, the electron impact ionization rate is neglected due to its smallness. Thus, the ionization rate of the hydro- 
gen atom is described as $\beta=\beta_{\mathrm{ce}}+\beta_{\mathrm{ph}}$ where $\beta_{\mathrm{ce}}$ is the charge exchange ionization rate, and $\beta_{\mathrm{ph}}$ is the photoionization rate. Daily charge-exchange rates $\left(\beta_{\mathrm{ce}}\right)$ were calculated using daily in situ observation parameters provided by the OMNI-2 data (OMNI WEB, Goddard Space Flight Center, NASA; http://omniweb.gsfc.nasa.gov/ow.html) using the formula:

$$
\beta_{\mathrm{ce}}=\frac{n v \sigma_{\mathrm{ce}}(v)}{r^{2}}=\frac{F \sigma_{\mathrm{ce}}(v)}{r^{2}}
$$

where $v$ is the solar wind velocity, $n$ is the solar wind density, $F$ is the solar wind flux, $r$ is the distance from the Sun, and $\sigma_{\mathrm{ce}}(v)$ is the cross-section for charge exchange, which was calculated following Lindsay and Stebbings (2005) from the formula:

$$
\sigma_{\mathrm{ce}}(v)=\left(a_{1}-a_{2} \log E(v)\right)^{2}\left(1-e^{-\frac{a_{3}}{E(v)}}\right)^{4.5}
$$

where $E(v)$ is the projectile energy in keV. $a_{1}=4.15$, $a_{2}=0.531$, and $a_{3}=67.3$ are coefficient parameters. Thus, $\sigma_{\mathrm{ce}}(v)$ is a function of the relative velocity between the atoms and the solar wind protons. The photoionization rate $\beta_{\mathrm{ph}}$ was derived from measurements made with the solar extreme ultraviolet monitor (SEM) on board the SOHO spacecraft at wavelengths of $0.1-50 \mathrm{~nm}$ (see http://www.spaceunibe.ch/soho/data.html). The spectral profile of solar EUV radiation (0.1-91.2 nm) was calculated using the SOLAR2000, and the cross-section for photoionization was calculated following the method of Cruddace et al. (1974). The photoionization rate in the ecliptic plane is then given by

$$
\beta_{\mathrm{ph}}=\int_{0}^{91.2} F_{\lambda} \sigma_{\mathrm{ph}} d \lambda
$$

where $\sigma_{\mathrm{ph}}(v)$ is the cross-section for photoionization, $F_{\lambda}$ is spectral flux, and $\lambda$ is wavelength. Figure 7 shows the variations in the rate of ionization for the period from March 1999 to April 2002. It is assumed that all of the variations in the factors affecting the ionization rate propagate instantaneously. While such an assumption is fully justified in the case of photoionization, it is not fully accurate in the case of the charge-exchange process (it takes approximately 1 year for a disturbance in the solar wind to propagate from $1 \mathrm{AU}$ to the termination shock). Since, however, the maximum of the backscattered Lyman $\alpha$ signal comes from about 3$5 \mathrm{AU}$ from the Sun and since most of the ionization losses of neutral interstellar hydrogen occur inside $\sim 10$ AU from the Sun, where the solar wind propagation time does not exceed $\sim 1.5$ months, the assumption of instantaneous propagation does not introduce serious discrepancies.

The backscatter intensities were calculated using the optically thin, single-scattering approximation, which means neglecting multiple scattering of the Lyman $\alpha$ photons on the heliospheric gas atoms (Quémerais, 2000). The solar illuminating flux is by no means spherically symmetric, and it fluctuates on time scales comparable to the solar rotation period. Hence, the non-radial LOS of Nozomi/UVS cannot be assumed to be illuminated by a spherically symmetric flux. The scale of the fluctuations can be observed in the

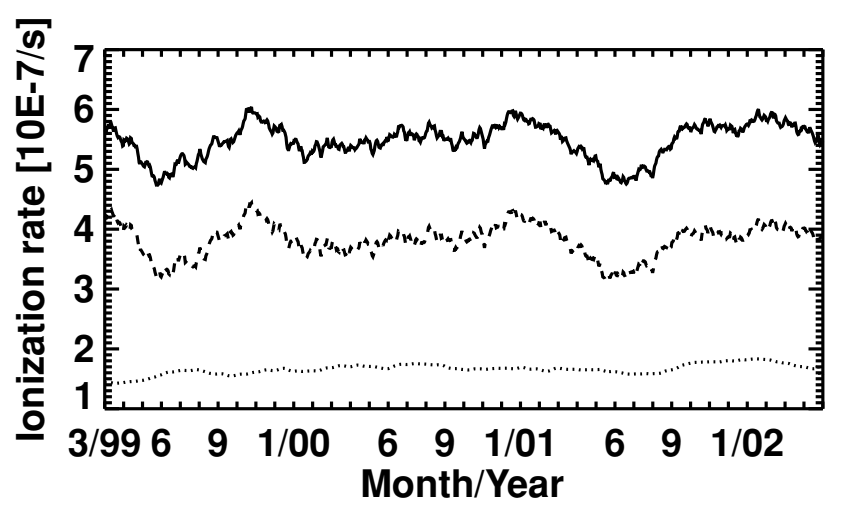

Fig. 7. Time variations in the rate of total ionization rate (solid), charge exchange ionization rate (broken line), and photoionization rate (dotted line) in the ecliptic plane used in the hot model for the period from March 1999 to April 2002.

SOLAR2000 time series, which was used to derive the normalized radiation pressure, as shown in Fig. 6.

Latitudinal asymmetries of the solar flux in the far ultraviolet (FUV), and H Lyman $\alpha$ were discussed in Cook et al. (1981), Pryor et al. (1992), and Auchère et al. (2005a, b). According to Cook et al. (1981), the latitudinal structure of the solar EUV irradiance can be approximated by the following function:

$$
F_{\mathrm{s}}(\alpha)=F_{\mathrm{s} \perp}+\left(F_{\mathrm{s} \|}-F_{\mathrm{s} \perp}\right) \cos ^{2} \alpha
$$

where $F_{\mathrm{s} \|}$ and $F_{\mathrm{s} \perp}$ are the solar Lyman $\alpha$ flux within the ecliptic plane and over the Sun's poles, respectively, and $\alpha$ is the solar equatorial latitude. Although the latitudinal structure according to Cook et al. (1981) describes the photon flux in the FUV region, we extend the same structure to the solar H Lyman $\alpha$ flux because the anisotropy of the $\mathrm{H}$ Lyman $\alpha$ solar flux has not yet been explored. The flux ratio of the solar H Lyman $\alpha$ above the ecliptic plane to over the pole is assumed to be 0.8 , which was estimated by Pryor et al. (1992) at the solar maximum period. Coincidentally, the photoionization rate is assumed to have the same anisotropy (i.e., 0.8).

\section{Latitudinal Anisotropy of the Ionization Rate}

The most important feature of this study is that the latitudinal anisotropy of the ionization rate of the chargeexchange is the only free parameter that is proportional to the solar wind proton flux and the cross section for chargeexchange, as shown in Eq. (1). Hence, if the cross section is known, the solar wind proton flux can be derived from the ionization rate. Summanen (1996) suggested the following formula to describe the ionization rate $\beta(b, r)$, which depends on an anisotropy constant $A_{\mathrm{c}}$ :

$$
\begin{aligned}
& \beta(b, r)=\beta_{0}\left(1-A_{\mathrm{c}}(\sin (c b))^{2}\right) \frac{r_{\mathrm{e}}^{2}}{r^{2}} \quad\left(-40^{\circ} \leq b \leq 40^{\circ}\right) \\
& \beta(b, r)=\beta_{0}\left(1-A_{\mathrm{c}}\right) \frac{r_{\mathrm{e}}^{2}}{r^{2}} \quad\left(|b| \geq 40^{\circ}\right)
\end{aligned}
$$

where $\beta_{0}$ is the ionization rate at $1 \mathrm{AU}$ from the Sun and at the equator in heliographic latitude, $r_{\mathrm{e}}$ is $1 \mathrm{AU}, A_{\mathrm{c}}$ is an 


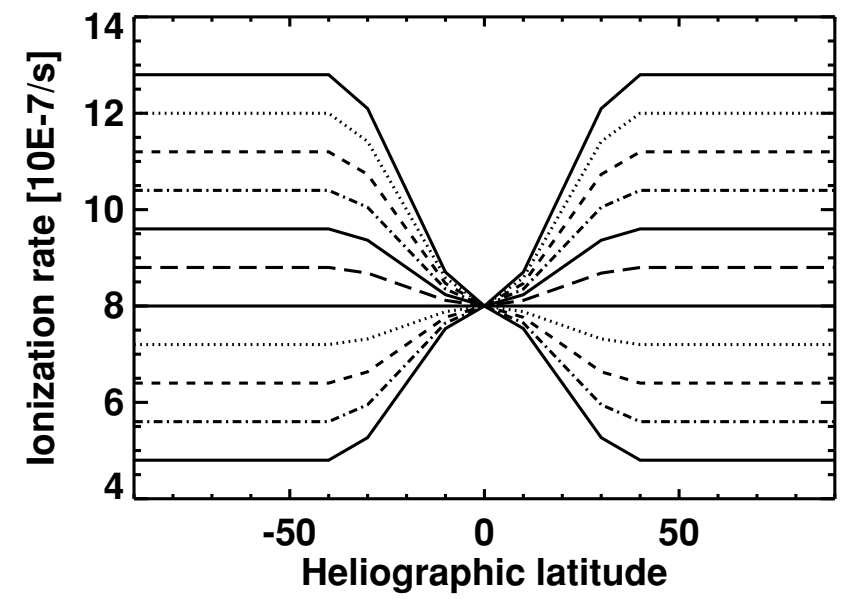

Fig. 8. Assumed latitudinal dependence of the ionization rate on the ionization rate for $A_{\mathrm{c}}=-0.6,-0.5,-0.4,-0.3,-0.2,-0.1,0.0$, $0.1,0.2,0.3$, and 0.4 from the upper line to the lower line when the ionization rate at the equator is assumed to be $8.0 \times 10^{-7} / \mathrm{s}$.

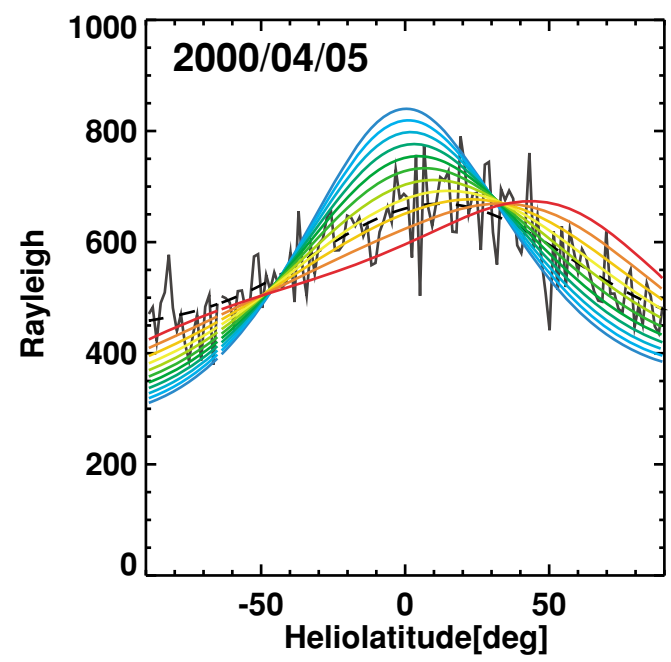

Fig. 9. The calculated intensity profiles of interplanetary Lyman $\alpha$ emission with multiple anisotropy of the ionization rate (color lines) are compared with the observational intensity profile obtained for April 5, 2000, corresponding to Fig. 4 (black line). Note that the data in the upwind hemisphere is shown. $A_{\mathrm{c}}=-0.6,-0.5,-0.4,-0.3,-0.2,-0.1,0.0$, $0.1,0.2,0.3$, and 0.4 correspond to the shift in the color of the lines from blue to red.

anisotropy constant, $c=9 / 4$ is a scaling factor for a smooth connection at $40^{\circ}$, and $r$ is the distance from the Sun. Previous studies have shown that $A_{\mathrm{c}}$ depends on the solar activity phase (Kumar and Boardfoot, 1979; Ajello et al., 1994; Pryor et al., 2001). In the case of $A_{\mathrm{c}}=0.4$, interplanetary hydrogen atoms at the equator are more ionized than at the poles. The case of $A_{\mathrm{c}}=0.0$ corresponds to an ionization rate that is independent of the ecliptic latitude. A wider range of $A_{\mathrm{c}}$ is allowed in our calculations than in those of Summanen (1996), who adopted values of between 0.0 and 0.4 . We investigated negative $A_{\mathrm{c}}$, which corresponds to the case of interplanetary hydrogen atoms at the poles being more ionized than those at the equator. Overall, we evaluated values of $A_{\mathrm{c}}$ between -0.6 and 0.4 in steps of 0.1 . Figure 8 shows the assumed latitudinal dependences of the ionization rate for the 11 values of $A_{\mathrm{c}}$ studied: $-0.6,-0.5$,
$-0.4,-0.3,-0.2,-0.1,0.0,0.1,0.2,0.3$, and 0.4 ; these correspond to the polar ionization rate normalized by the equatorial ionization rate $R=1-A_{\mathrm{c}}$ of $1.6,1.5,1.4,1.3$, $1.2,1.1,1.0,0.9,0.8,0.7$, and 0.6 , respectively. We derived the intensity of the interplanetary hydrogen emission using the calculated ionization rate for these 11 cases of $A_{\mathrm{c}}$. The calculated intensity profile is shown in Fig. 9 compared with the intensity profile observed for April 5, 2000.

\section{Chi-Square Fit}

The calculated intensities are normalized by the observed average as follows:

$$
I_{\mathrm{cal}}=I_{\mathrm{cal}} \times\left(\frac{\sum_{i=1}^{N} I_{\mathrm{obs}}}{\sum_{i=1}^{N} I_{\mathrm{cal}}}\right)
$$

where $N$ is the number of data points for each day ( $N=$ 256) and $I_{\mathrm{cal}}$ and $I_{\mathrm{obs}}$ are the calculated intensity and observational intensity, respectively, for each LOS. Model calculations for various values of $A_{\mathrm{c}}$ are compared with the UVS data using the residual $\chi^{2}$, which is defined as

$$
\begin{aligned}
\chi^{2} & =\sum_{1}^{N} \frac{\left(I_{\mathrm{obs}, i}-C I_{\mathrm{cal}, i}\right)^{2}}{\sigma_{i}^{2}} \\
C & =\frac{1}{N} \sum_{i}^{N} \frac{I_{\mathrm{obs}, i}}{I_{\mathrm{cal}, i}}
\end{aligned}
$$

where $\sigma$ is the root mean square (RMS) deviation of $I_{\mathrm{obs}}$, and $C$ is a scaling factor. The values of $\chi^{2}$ are computed for the 11 cases, and the value of $A_{\mathrm{c}}$ corresponding to the smallest $\chi^{2}$ is adopted as the best fit solution. Finally, the latitudinal distribution of the ionization rate is computed for each day. To evaluate the procedure, we compared the observed and calculated Lyman $\alpha$ intensities; examples on April 5, 2000, corresponding to Fig. 9, are shown in Fig. 10. Note that the $\chi^{2}$ analysis is performed for data taken only in the upwind hemisphere. In Fig. 10, $\chi^{2}$ values

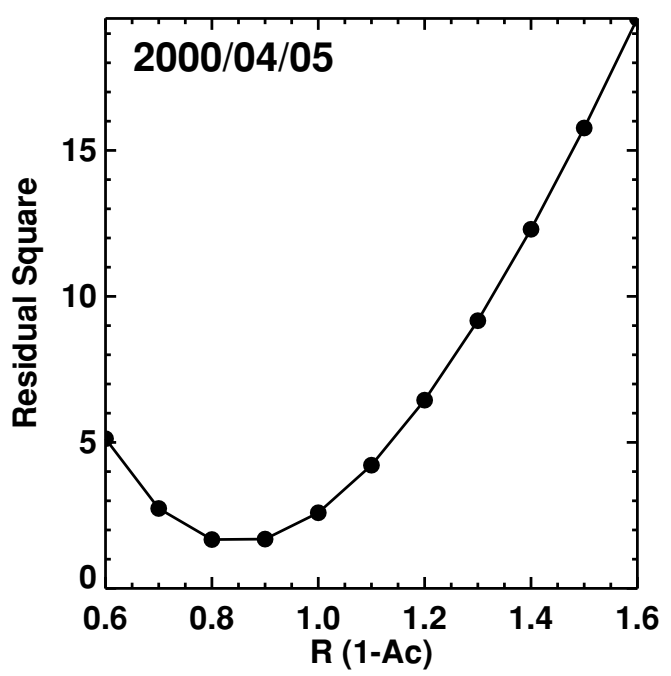

Fig. 10. Example of comparisons between observed intensity profile and calculated intensities. $\chi^{2}$ values corresponding to $A_{\mathrm{c}}=-0.6,-0.5$, $-0.4,-0.3,-0.2,-0.1,0.0,0.1,0.2,0.3$, and $0.4\left(R\left(=1-A_{\mathrm{c}}\right)=1.6\right.$, $1.5,1.4,1.3,1.2,1.1,1.0,0.9,0.8,0.7$, and 0.6$)$ are shown. 
corresponding to the 11 cases for $A_{\mathrm{c}}$ (or $R$ ) are shown by filled circles. For reference (i.e., April 5, 2000 observation), the value of $R\left(1-A_{\mathrm{c}}\right)$ was determined to be 0.8 .

\section{Determination of Solar Wind Density}

Once the anisotropy of the ionization rate $A_{\mathrm{c}}$ is determined, the anisotropy of the total ionization rate $\beta(b, r)$ and that of the charge exchange ionization rate $\beta_{\text {ce }}$ can be derived, due to the assumption of photoionization rate $\beta_{\mathrm{ph}}$. The latitudinal dependence of the solar wind velocity is needed to derive the latitudinal dependence of the solar wind proton flux and density using the charge exchange ionization rate $\beta_{\mathrm{ce}}$, as shown in Eq. (1). The IPS observations conducted by the STEL of Nagoya University (Kojima and Kakinuma, 1990; Kojima et al., 1998) were used to obtain the solar wind velocity $v$ (and hence the cross-section of the charge-exchange $\left.\sigma_{\text {ce }}(v)\right)$ in Eq. (1). This enabled accurate determination of the global distribution of the solar wind speed. The computer-assisted tomography method is employed to obtain the global distribution of the speed data from the STEL IPS observations.

\section{Results}

The amplitude of the ionization rate anisotropy and its variation for 3 years from 1999 to 2002 are shown in Fig. 11. The variation in both hemispheres was mostly similar, except for the enhancement of the flux in the southern hemisphere on June, 1999. The shift from an anisotropic ionization rate pattern at solar minimum to an isotropic pattern at solar maximum was clearly visible. Although the ionization rate at the equator was larger than that at the pole before the middle of 2000, the polar-to-equatorial ionization rate ratio increased toward the solar maximum, and the latitudinal dependence of the ionization rate roughly shows an isotropy. During this period of solar cycle, fast and slow solar winds are mixed at all latitudes. Thus, the ionization rate-by-charge exchange with the solar wind proton is mostly constant with heliographic latitude. Within the margin of statistical error, the polar ionization rate did not significantly exceed the equatorial ionization rate even in the solar maximum period. Once the ionization rate ratio was far below $R=1$ in the last half of 2001, its ratio began to increase again from the beginning of 2002 onwards. This behavior is very similar to the activity of the solar cycle, as represented by the variation of the solar Lyman $\alpha$ flux or radiation pressure in Fig. 6. The polar ionization rate normalized by the equatorial ionization rate correlates well with solar cycle activity.

Using the ionization rate data, we derived the solar wind proton flux from 1999 to 2002 . These data are plotted in a latitude versus time diagram in Fig. 12 (top). The solar wind speed derived from STEL observations and the solar wind density determined by our method are also shown in Fig. 12 (middle and bottom, respectively). It is evident from Fig. 12 that we successfully observed the slow and dense solar wind over the poles during the solar maximum period. The polar solar wind density indicates a significant growth from the first half of 2000 in both hemispheres, and the polar values of solar wind density are close to the equatorial values. This result is due to the disappearance of the coronal

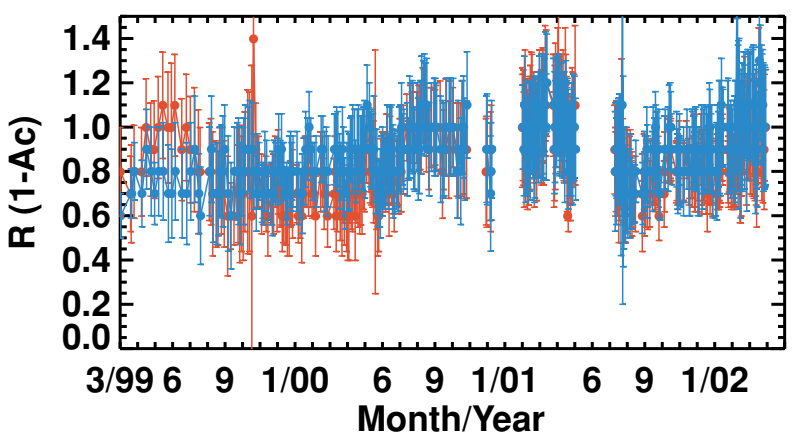

Fig. 11. Amplitude of the ionization rate anisotropy and its variation. The blue lines show the variation of the asymmetry of the ionization rate in the northern hemisphere and the red lines show the same in the southern hemisphere. The vertical axis represents the polar-to-equatorial ionization rate ratio. The horizontal axis represents the month in the period from March 1999 to April 2002.
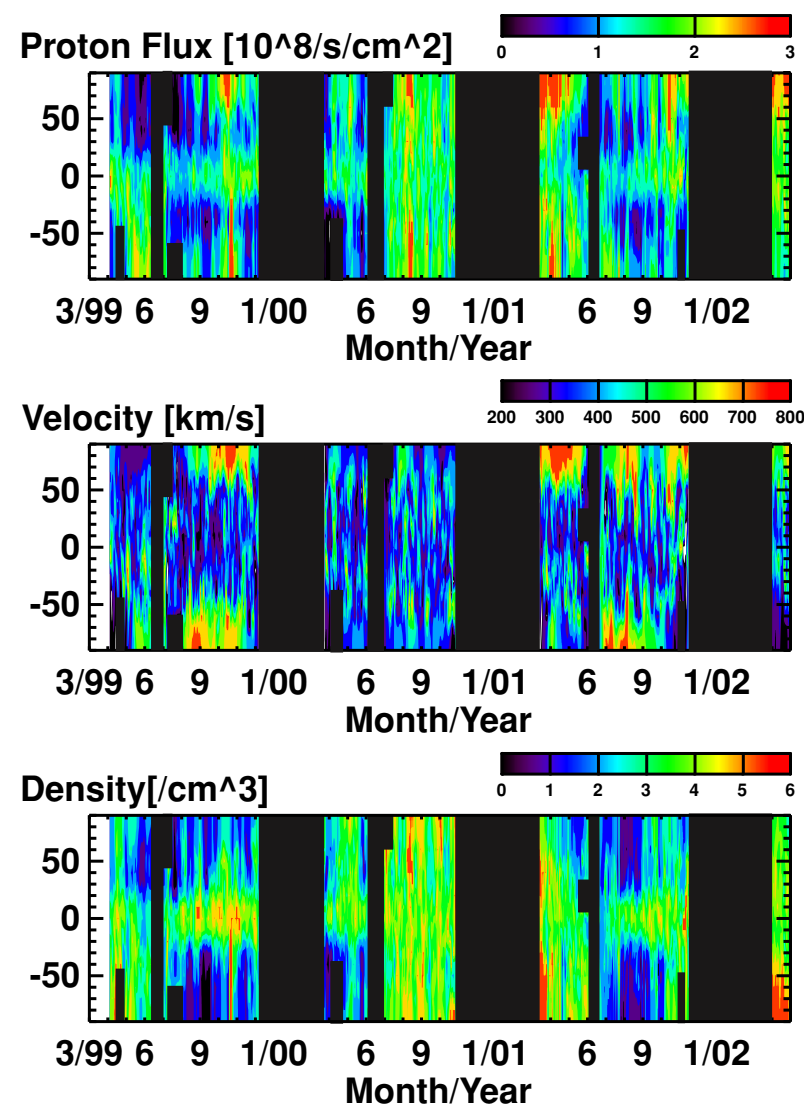

Fig. 12. A latitude-vs.-time diagram of the solar wind speed (middle) derived from STEL observations, solar wind proton flux (top), and density (bottom) made by our method.

hole. In addition, a marked density depletion occurs in the middle of 2001 in both hemispheres, which is ascribed to the development of fast winds from the polar coronal hole. After 2002, the polar solar wind density increased again due to the disappearance of the polar coronal hole.

\section{Discussion}

We have shown how the latitudinal dependence of the ionization rate and solar wind changes during the solar maximum period. The variation in the latitudinal depen- 


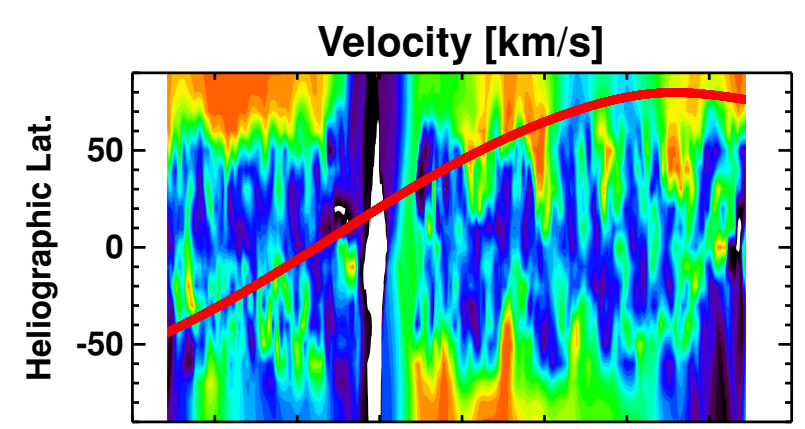

Mar. Apr. Jun. Jul. Aug. Sep. Oct. Nov.

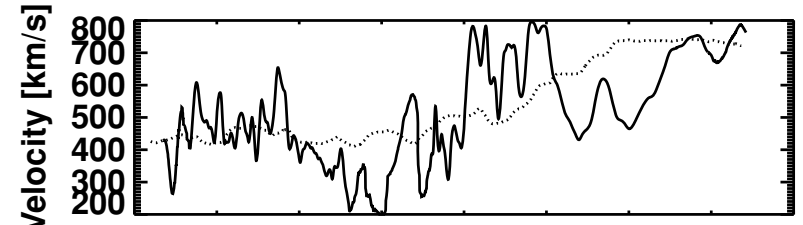

Mar. Apr. Jun. Jul. Aug. Sep. Oct. Nov.

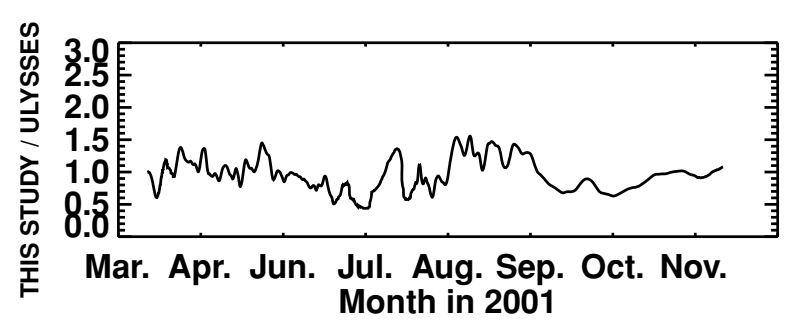

Fig. 13. Comparisons between the solar wind speed derived from STEL observations and that measured by the Ulysses in 2001. The top three panels correspond to latitude-vs.-time diagrams of the solar wind speed derived from STEL observations. The red lines represent the trajectory of the Ulysses spacecraft. The middle panels show the time variation of the solar wind speed density measured along the trajectory of Ulysses. The broken and solid lines represent the Ulysses measurements and STEL observations, respectively. The bottom panels show the ratio of STEL observations divided by Ulysses measurements for the solar wind speed. The horizontal axis indicates the month in 2001 and the vertical axis represents the heliographic latitude.

dence of the ionization rate shown in Fig. 11 is basically similar to that reported previously (Nakagawa et al., 2003; Quémerais et al., 2006). Quémerais et al. (2006) derived the latitudinal dependence of the total ionization rate $\beta_{0}$ from the SOHO/SWAN measurements during a period of almost 10 years (1996-2006). Their results suggest that the ionization was strongly asymmetric between 1996 and 2000, with an excess of $70 \%$ in 1998 , and that the ionization rate was roughly symmetrical between the last half of 2000 and 2004 , although even in this period the equatorial ionization rate was still slightly larger than the pole values. These results have a good consistency with our results in Fig. 11.

The solar wind density data derived from this study are compared with in situ measurements by the Ulysses spacecraft from 2001, with the spacecraft moving from the southern high latitudes to the northern poles (a so-called "fast scan"). Figs. 13-15 shows comparisons between our measurements and those of Ulysses. The top panels of Figs. 1315 correspond to the latitudinal-vs.-time diagram of the solar wind speed derived from STEL observations, proton flux, and density derived from our method. The red line in each plot represents the trajectory of the Ulysses spacecraft.

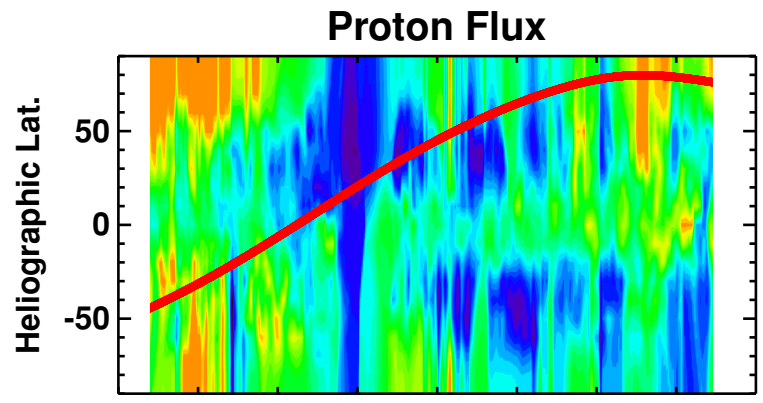

Mar. Apr. Jun. Jul. Aug. Sep. Oct. Nov.
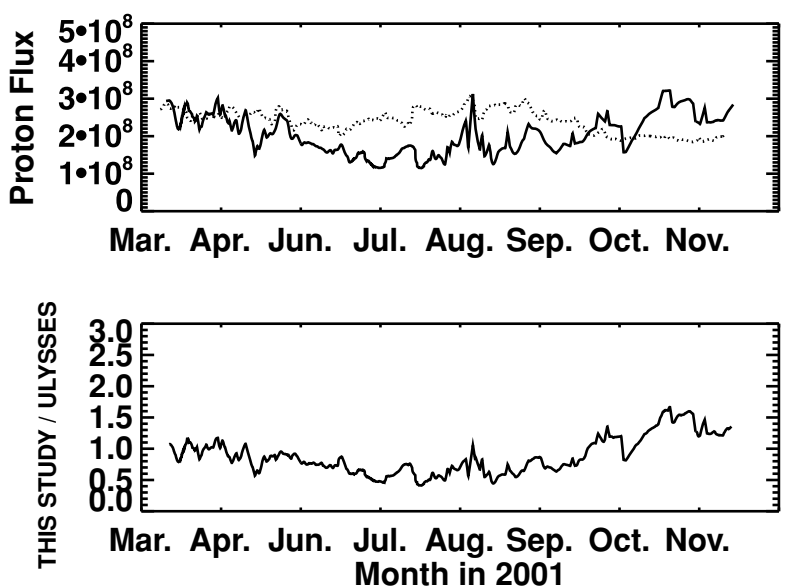

Fig. 14. Comparisons between the solar wind proton flux made by our method and that measured by the Ulysses spacecraft in 2001. The format is the same as that in Fig. 13.

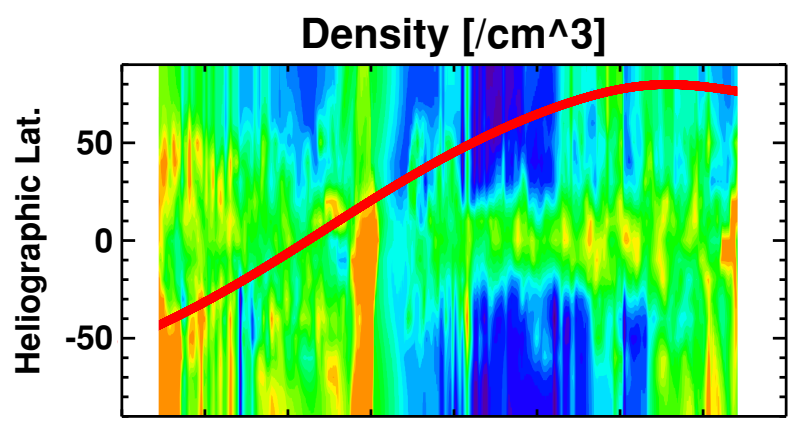

Mar. Apr. Jun. Jul. Aug. Sep. Oct. Nov.

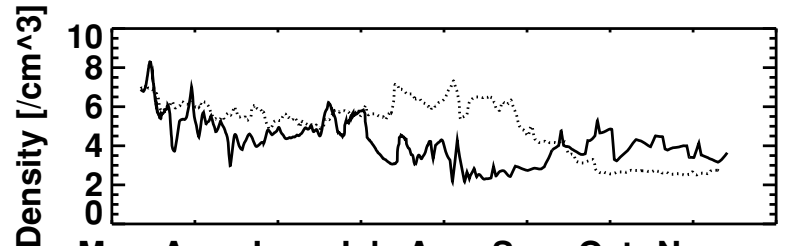

Mar. Apr. Jun. Jul. Aug. Sep. Oct. Nov.
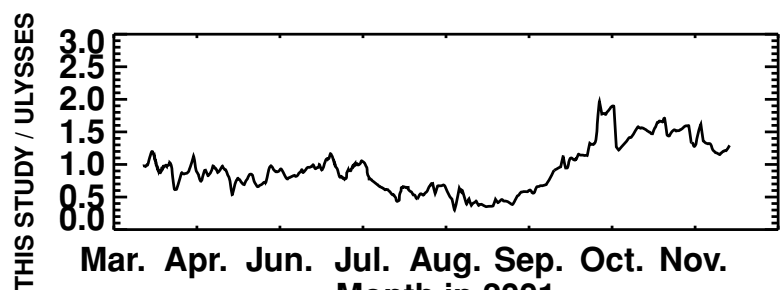

Mar. Apr. Jun. Jul. Aug. Sep. Oct. Nov. Month in 2001

Fig. 15. Comparisons between the solar wind density made by our method and that measured by the Ulysses spacecraft in 2001. The format is the same as that of Fig. 13. 
The middle panels show the time variation of the solar wind speed, proton flux, and density measured along the trajectory of Ulysses. The Ulysses' values are smoothed by the 27-day running mean method. The broken and solid lines in the middle panels represent the values of Ulysses and those of our method, respectively. The bottom panels show the ratios of our method divided by Ulysses measurements for the solar wind speed, proton flux, and density. The density data obtained in this study basically show a good agreement with the Ulysses data in a long-term trend. However, our density data were systematically smaller than the Ulysses data by a factor of approximately 2 from July to September in 2001. Meanwhile, note that our method overestimated both the density and the proton flux in the high latitudinal region. One possible reason for this overestimation is that in a highly variable phase of the solar activity the solar wind structure is unstable in the solar maximum phase. Moreover, the Ulysses' values reflect local conditions in the solar wind, whereas our method reflects relatively global conditions. The influence of coronal mass ejections (CMEs) may also cause this difference. The effect of CMEs is neglected in this study; it is necessary to explore CMEs in future investigations.

The main uncertainty in the analysis methods used here can be considered to originate from the ambiguity of the parameters assumed in the model calculations, such as the radiation pressure, the ionization rate, the solar Lyman $\alpha$ flux at all latitudes, and the density of the hydrogen atom. Quémerais et al. (2006) reported that ionization rates derived from in situ solar wind measurements are significantly smaller than their results from interplanetary Lyman $\alpha$ measurements at solar maximum. This discrepancy remains an open question. A more accurate estimation of these parameters would be beneficial, although such a study is beyond the scope of this work. We assume that a classical hot model is appropriate for this work as a first step to a separate evaluation of the remote sensing method and model. A more realistic model and the dynamic behavior on a much shorter time scale can be investigated as the next step in future work using time-dependent heliospheric models, such as those of Rucinski and Bzowski (1995), Zank (1999), Fahr et al. (2000), Müller et al. (2004), Pogorelov et al. (2004), and Izmodenov et al. (2008).

\section{Conclusions}

In this study, the interplanetary Lyman $\alpha$ emission observations of the Nozomi spacecraft are analyzed with the aim of investigating the latitudinal variation of the ionization rate of interplanetary hydrogen and its long-term evolution during 1999-2002. The results show that the polar ionization rate increases during the ascending phase and is almost equivalent with the equatorial ionization rate at the solar maximum, which coincides with previous results. We successfully observed the slow and dense solar wind over the poles during solar maximum period. Our calculations of polar solar wind density indicates that it experienced a significant growth from the middle of 2000 onwards. In addition, a marked density depletion occurred in the middle of 2001 , and this is ascribed to the development of fast winds from the polar coronal hole. A comparison with Ulysses in situ measurements suggests a roughly consistent—although significant (a factor of 2)-difference between the Ulysses measurements and our measurements in the middle of 2001. The cause of this discrepancy is not understood and requires further exploration in a future study.

Acknowledgments. The authors wish to thank the SolarTerrestrial Environment Laboratory of Nagoya University for allowing us to use the interplanetary scintillation (IPS) data. We would like to thank Dr. M. Tokumaru for their valuable comments regarding this paper. We would also like to thank the SOHO/SEM, Ulysses, and OMNI WEB team members for their valuable data. Data from the OMNI WEB and Ulysses spacecraft data were provided by NSSDC. The use of SOLAR2000 software for the calculations is also gratefully acknowledged. This work was supported by the Scientific Research Fund of Japan Society for the Promotion of Science (JSPS). This work was carried out by the joint research program of the Solar-Terrestrial Environment Laboratory, Nagoya University.

\section{References}

Ajello, J. M., W. R. Pryor, C. A. Barth, C. W. Hord, A. I. F. Stewart, K. E. Simmons, and D. T. Hall, Observations of interplanetary Lyman- $\alpha$ with the Galileo Ultraviolet Spectrometer: multiple scattering effects at solar maximum, Astron. Astrophys., 289, 283, 1994.

Auchère, F., Effect of the HI Ly $\alpha$ chromospheric flux anisotropy on the total intensity of the resonantly scattered coronal radiation, Astrophys. $J ., 622,737,2005$ a.

Auchère, F., J. W. Cook, J. S. Newmark, D. R. McMullin, R. von Steiger, and M. Witte, The heliospheric HeII $30.4 \mathrm{~nm}$ solar flux during cycle 23, Astrophys. J., 625, 1036, 2005 b.

Baranov, V. B., ISSI Sci Rep., SR-005, 1, 2006.

Bertaux, J. L., E. Kyrölä, E. Quémerais, R. Pellinen, R. Lallement, W. Schmidt, M. Berthe, E. Dimarellis, J. P. Goutail, C. Taulemesse, C. Bernaud, G. Leppelmeier, T. Summanen, H. Hannula, H. Huomo, V. Kehla, S. Korpela, K. Leppala, E. Strommer, J. Torsti, K. Viherkanto, J. F. Hochedez, G. Chretiennot, R. Peyroux, and T. Holzer, SWAN: A study of solar wind anisotropies on SOHO with Lyman $\alpha$ sky mapping, Solar Phys., 162, 403, 1995.

Bzowski, M., E. Möbius, S. Tarnopolski, V. Izmodenov, and G. Gloeckler, Density of neutral interstellar hydrogen at the termination shock from Ulysses pickup ion observations, Astron. Astrophys., 491, 7, 2008.

Cook, J. W., R. Meier, G. E. Brueckner, and M. E. VanHoosier, Latitudinal anisotrophy of the solar far ultraviolet flux: effect on the L $\alpha$ sky background, Astron. Astrophys., 97, 394, 1981.

Cruddace, R., F. Paresce, S. Bowyer, and M. Lampton, On the opacity of the interstellar medium to ultrasoft X-rays and extreme-ultraviolet radiation, Astrophys. J., 187, 497, 1974.

Fahr, H. J., Change of interstellar gas parameters in stellar-wind-dominated astrospheres: solar case, Astron. Astrophys., 66, 103, 1978.

Fahr, H. J., The interstellar gas flow through the heliospheric interface region, Space Sci. Rev., 78, 199, 1996.

Fahr, H. J., T. Kausch, and H. Scherer, A 5-fluid hydrodynamic approach to model the solar system-interstellar medium interaction, Astron. Astrophys., 357, 268, 2000.

Fukunishi, H., S. Watanabe, M. Taguchi, and Y. Takahashi, Mars ultraviolet imaging spectrometer experiment on the PLANET-B mission, Adv. Space Res., 23, 1903, 1999.

Gloeckler, G. and J. Geiss, Heliospheric and interstellar phenomena deduced from pickup ion observations, Space Sci.Rev., 97, 169, 2001.

Izmodenov, V., The Sun and the heliosphere as an integrated system, Kluwer Academic Publishers, 2004.

Izmodenov, V. and V. B. Baranov, ISSI Sci. Rep., SR-005, 67, 2006.

Izmodenov, V., Y. G. Malama, and M. S. Runderman, Modeling of the outer heliosphere with the realistic solar cycle, Adv. Space Res., 41, 318, 2008.

Joselyn, J. A. and T. E. Holzer, The effect of asymmetric solar wind on the Lyman alpha sky background, J. Geophys. Res., 80, 903, 1975.

Kojima, M. and T. Kakinuma, Solar cycle evolution of solar wind speed structure between 1973 and 1985 observed with the interplanetary scintillation method, J. Geophys. Res., 92, 7269, 1987.

Kojima, M. and T. Kakinuma, Solar cycle dependence of global distribution of solar wind speed, Space Sci.Rev., 53, 173, 1990. 
Kojima, M., M. Tokumaru, H. Watanabe, and A. Yokobe, Heliospheric tomography using interplanetary scintillation observations 2. Latitude and heliocentric distance dependence of solar wind structure at $0.1-$ 1 AU, J. Geophys. Res., 103, 1981, 1998.

Kumar, S. and A. L. Broadfoot, Signatures of solar wind latitudinal structure in interplanetary Lyman- $\alpha$ emissions: MARINER 10 observations, Astrophys. J., 228, 302, 1979.

Kyrörä, E., T. Summanen, W. Scmidt, T. Mäkinen, E. Quémerais, J. L. Bertaux, R. Lallement, and J. Costa, Preliminary retrieval of solar wind latitude distribution from solar wind anisotropies/SOHO observations, J. Geophys. Res., 103, 14523, 1998.

Lallement, R., J. L. Bertaux, and F. Dalaudier, Interplanetary Lyman $\alpha$ spectral profiles and intensities for both repulsive and attractive solar force fields: predicted absorption pattern by a hydrogen cell, Astron. Astrophys., 150, 21, 1985.

Lallement, R., E. Quémerais, J. L. Bertaux, S. Ferron, D. Koutroumpa, and R. Pellinen, Deflection of the interstellar neutral hydrogen flow across the heliospheric interface, Science, 307, 1447, 2005.

Lindsay, B. G. and R. F. Stebbings, Charge transfer cross sections for energetic neutral atom data analysis, J. Geophys. Res., 110, A12213, doi:10.1029:2005JA011298, 2005.

Marsden, R. G., E. J. Smith, J. F. Cooper, and C. Tranquille, ULYSSES at high heliographic latitudes: an introduction, Astron. Astrophys., 316, 279, 1996.

McComas, D. J., H. A. Elliott, and R. von Steiger, Solar wind from highlatitude coronal holes at solar maximum, Geophys. Res. Lett., 29, 1314, 2002.

McComas, D. J., H. A. Elliott, N. A. Schwadron, J. T. Gosling, R. M. Skoug, and B. E. Goldstein, The three-dimensional solar wind around solar maximum, Geophys. Res. Lett., 30, 1517, 2003.

Müller, H.-R. and G. P. Zank, Heliospheric filtration of interstellar heavy atoms: Sensitivity to hydrogen background, J. Geophys. Res., 109, A07104, 2004.

Nakagawa, H., H. Fukunishi, Y. Takahashi, S. Watanabe, M. Taguchi, J. L. Bertaux, R. Lallement, and E. Quémerais, Solar cycle dependence of interplanetary Lyman $\alpha$ emission and solar wind anisotropies derived from NOZOMI/UVS and SOHO/SWAN observations, J. Geophys. Res., 108, 8035, 2003.

Phillips, J. L., S. J. Bame, A. Barnes, B. L. Barraclough, W. C. Feldman, B. E. Goldstein, J. T. Gosling, G. W. Hoogeveen, D. J. McComas, M. Neugebauer, and S. T. Suess, Ulysses solar wind plasma observations from pole to pole, Geophys. Res. Lett., 22, 3301, 1995.

Pogorelov, N. V., G. P. Zank, and T. Ogino, Three-dimensional features of the outer heliosphere due to coupling between the interstellar and interplanetary magnetic fields. 1. Magnetohydrodynamic model: Interstellar perspective, Astrophys. J., 614, 1007, 2004.

Pryor, W. R., J. M. Ajello, C. A. Barth, C. W. Hord, A. I. F. Stewart, K. E. Simmons, W. E. McClintock, B. R. Sandel, and D. E. Shemansky, The Galileo and Pioneer Venus ultraviolet spectrometer experiments: solar Lyman- $\alpha$ observations, Astrophys. J., 394, 363, 1992.

Pryor, W. R., I. Stewart, K. Simmons, M. Witte, J. Ajello, K. Toskiba, D. McComas, and D. Hall, Remote sensing of $\mathrm{H}$ from Ulysses and Galileo, Space Sci. Rev., 97, 393, 2001.

Pryor, W. R., J. M. Ajello, D. J. McComas, M. Witte, and W. K. Tobiska, Hydrogen atom lifetimes in the three-dimensional heliosphere over the solar cycle, J. Geophys. Res., 108, 8034, 2003.

Quémerais, E., Angle dependent partial frequency redistribution in the interplanetary medium at Lyman $\alpha$, Astron. Astrophys., 358, 353, 2000.

Quémerais, E., R. Lallement, S. Ferron, D. Koutroumpa, J. L. Bertaux, E. Kyrölä, and W. Schmidt, Interplanetary hydrogen absolute ionization rates: Retrieving the solar wind mass flux latitude and cycle dependence with SWAN/SOHO maps, J. Geophys. Res., 111, A09114, doi:10.1029/2006JA011711, 2006.

Rucinski, D. and M. Bzowski, Modulation of interplanetary hydrogen density distribution during the solar cycle, Astron. Asrophys., 296, 248, 1995.

Summanen, T., The effect of the time and latitude-dependent solar ionization rate on the measured Lyman $\alpha$-intensity, Astron. Astrophys., 314, 663, 1996.

Summanen, T., The solar ionization rate of the interplanetary hydrogen as a function of a heliomagnetic latitude: a new model for the interplanetary Lyman alpha studies, Astrophys. Space Sci., 274, 143, 2000.

Taguchi, M., H. Fukunishi, S. Watanabe, S. Okano, Y. Takahashi, and T. D. Kawaahara, Ultraviolet imaging spectrometer (UVS) experiment on board the NOZOMI spacecraft: Instrumentation and initial results, Earth Planets Space, 52, 49, 2000.

Thomas, G. E., The interstellar wind and its influence on the interplanetary environment, Ann. Rev. Earth Planet. Sci., 6, 173, 1978.

Tobiska, W. K., T. Woods, F. Eparvier, R. Viereck, L. Floyd, D. Bouwer, G. Rottman, and O. R. White, The SOLAR2000 empirical solarirradiance model and forecast tool, J. Atmos. Sol.-Terr. Phys., 81, 1247, 2000.

Wu, F. M. and D. L. Judge, Temperature and flow velocity of the interplanetary gases along solar radii, Astrophys. J., 231, 594, 1979.

Zank, G. P., Interaction of the solar wind with the local interstellar medium: a theoretical perspective, Space Sci. Rev., 89, 413, 1999.

H. Nakagawa (e-mail: rom@stpp1.geophys.tohoku.ac.jp), H. Fukunishi, S. Watanabe, Y. Takahashi, M. Taguchi, and R. Lallement 